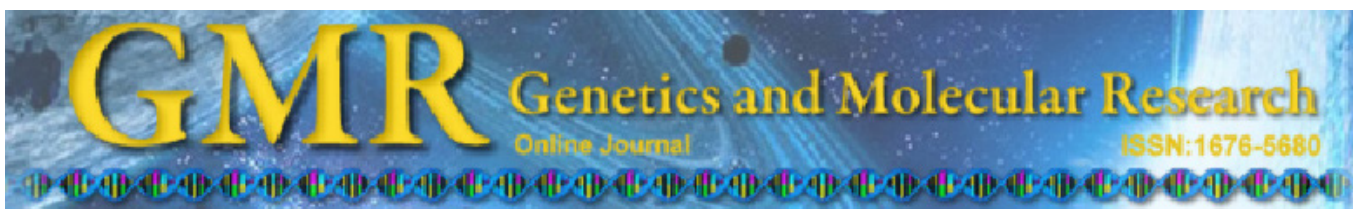

$\underline{\text { Short Communication }}$

\title{
"Extended Fitness" hypothesis: a link between individual and group selection
}

\author{
S.J. de Souza \\ Instituto do Cérebro, Universidade Federal do Rio Grande do Norte, \\ Natal, RN, Brasil \\ Corresponding author: S.J. de Souza \\ E-mail: sandro@neuro.ufrn.br
}

Genet. Mol. Res. 12 (4): 4625-4629 (2013)

Received August 29, 2013

Accepted September 12, 2013

Published October 17, 2013

DOI http://dx.doi.org/10.4238/2013.October.17.5

\begin{abstract}
What are the targets of natural selection remains a controversial issue in Biology. Here I propose the "Extended Fitness" hypothesis, in which extended phenotypes emerge as a link between individual and group selection. The basic premise of the extended fitness hypothesis is that extended phenotypes can be used by members of the same group since they are adapted to use them. Thus, extended phenotypes can also contribute to the fitness of members of the same species. Group selection emerges as a natural consequence of the shared use of extended phenotypes, which allow the evolutionary forces acting on individuals to become effective at the group level. The extended fitness hypothesis is supported by several observations found in nature. The hypothesis presented here provides a theoretical framework for the design of both modeling and experimental approaches to studying the role of extended phenotypes in group selection.
\end{abstract}

Key words: Extended phenotypes; Group selection; Kin selection; Fitness 


\section{INTRODUCTION}

One of the most important debates in biology concerns the targets of natural selection. While Darwin put significant emphasis on individual selection, he also discussed that under some circumstances selection between groups could prevail even at the expense of withingroup selection (Darwin, 1859). Although the dominant view in the 1960 s was that individual selection was the rule and group selection was the exception, Hamilton, in 1964, proposed a mathematical model that could explain the selection of "altruistic" genetic variants, especially among kin, a concept he called "inclusive fitness" (Hamilton, 1964a,b). Fifteen years later, Dawkins (1976) proposed an ultra-Darwinian theory, called "Selfish Gene", in which genetic replicators would be the main targets of natural selection. The whole debate was reinvigorated in the last few years because of a publication by Nowak et al. (2010), where the authors claimed to have proved that "inclusive fitness" would be untenable. That specific report was strongly rebutted by more than one hundred authors (Abbot et al., 2011).

A key concept in this debate is the extended phenotype. According to Dawkins, a gene's action does not need to be restricted to the body of the individual organism where the gene is located (Dawkins, 1982). A gene can extend its effect such as in a bird's nest and the beaver's dam, the most known examples of extended phenotypes. While there is significant controversy over the impact of the extended phenotype within the contemporary evolutionary theory (Hunter, 2009), there is little doubt about its widespread existence in nature.

\section{THE EXTENDED FITNESS HYPOTHESIS}

While observing a couple of spiders close to a web in the garden of my workplace, I realized that extended phenotypes could serve other individuals of the same group or species. Accordingly, an extended phenotype would not only contribute to the fitness of the individual who generated it, but it would also have an impact on the fitness of individuals who can use and take advantage of it. Those individuals would be members of the same group since they are already adapted to use a similar extended phenotype. I call this conceptual framework, the "Extended Fitness" hypothesis. The basic premise of the extended fitness hypothesis is that a group of individuals who share some level of genetic similarity is under selection as a group through the use of each member's extended phenotypes. Only those individuals that share enough genetic similarity with the individual responsible for the generation of the extended phenotype will have the proper features to take full advantage of the extended phenotype. By "enough genetic similarity", I mean the level of genetic identity present in individuals from a given group who can use extended phenotypes from conspecifics since they are able to generate the same extended phenotype.

Several examples observed in nature support the extended fitness hypothesis. Extended phenotypes can be abandoned by individuals, which make them available to be used by other individuals of the same group. Several species of spiders for example abandon their webs and relocate to new sites (Schuck-Paim and Alonso, 2001). Such webs can then be used by other members of the same group. These other members would use the extended phenotype properly because of the genetic similarity with the individual that produced the extended phenotype. If, for example, crater-building cichlids are experimentally removed from a site, their crater is immediately taken-over by other members of the same species (Schaedelin and Ta- 
borsky, 2006). Another well-known example is the case of the digger wasp Sphex ichneumoneus. The wasp digs a burrow, fills it with dead insects and then lays an egg in it. The burrows can be abandoned and occupied by other individuals of the same species at a high frequency (Brockmann et al., 1979).

Also supporting the idea that extended phenotypes could serve as a basis for group selection is the fact that most of the extended phenotypes are species-specific. Under the extended fitness hypothesis, selection would favor groups that develop extended phenotypes that only their members can use. The horn-shaped subterranean burrows built by male mole crickets are extended phenotypes that are species-specific (Bailey, 2012). The morphology of oak galls built by gallwasps in their oak hosts is also species-specific (Bailey et al., 2009).

Research in the last decade has shown that in several species individuals prefer to settle in areas with a higher density of individuals of the same species. This has been referred to as conspecific attraction (Reed and Dobson, 1993). For example, the spider Nephilengys cruentata prefers to build webs in areas with a high density of conspecifics (Schuck-Paim and Alonso, 2001). Conspecific attraction only works if the benefits of settling with conspecifics out-weight the costs of a higher density of conspecifics. Two types of benefits have been proposed to explain conspecific attraction: reproduction effects and measurements of habitat quality. The extended fitness hypothesis can be another possible explanation for conspecific attraction. One efficient way for an individual to use the extended phenotype of other members of the same species is to be closer to them.

The extended fitness could act at different group levels. For example, it is reasonable to think that most members of a given species have the proper genetic background to take advantage of extended phenotypes generated by their conspecifics. On the other hand, it is also reasonable to think that genetic variants more frequently found in a given population, within a species, make members of that group more adapted to use each other's extended phenotypes. There is no need, however, for these members to be close relatives, which precludes the extended fitness to be classified as a type of kin selection.

\section{PREDICTIONS AND CONCLUSION}

A prediction of the extended fitness hypothesis is that species with a stronger social behavior would use more frequently extended phenotypes generated by other members of the same group. Indeed, this seems to be the case. Interactions between social insects, for example, depend heavily on extended phenotypes. For example, ants are known for marking a specific pathway on the ground with chemicals that signal to other members of the same species a track to follow. That track itself can be considered to be an extended phenotype (Schaedelin and Taborsky, 2009). Only members of the same species are able to recognize the chemicals in the track and to respond to them properly. The same is true for ant nests, another example of extended phenotype used for several individuals of the same group (Minter et al., 2012).

I believe that the extended fitness hypothesis provides a theoretical framework that links individual and group selection. A key issue for the validity of group selection is the assumed need for between-group selection to overcome, in terms of fitness, within-group selection. Since the extended phenotype contributes to the fitness of the individual who generates it, there is no need, under the extended fitness hypothesis, for this unbalanced selection to explain the emergence of selection at the group level. This effect at the group level is a natural conse- 
quence of the fact that individuals of the same group are already adapted to use each other's extended phenotypes. In a classical situation of between-group competition, groups with that capacity would out compete groups without that capacity.

One interesting aspect of the extended fitness hypothesis is its lower sensitivity to the emergence of "cheaters", in this case genetic variants that stop producing their own extended phenotypes and only use extended phenotypes of other individuals. While this is possible, the fitness of the "cheaters" will likely be less since they will depend exclusively on the availability of extended phenotypes generated by other individuals. This has been shown computationally (Xavier and Foster, 2007) as well as experimentally (Nadell and Bassler, 2011) in biofilms containing both altruistic and cheater variants of microbes. In spite of the cost of producing biofilm, altruistic producers outcompete cheaters due to their positions within the biofilm, which allow them to access nutrients more efficiently (Xavier and Foster, 2007; Nadell and Bassler, 2011). Another prediction of the extended fitness hypothesis is that besides a lower individual fitness for the extended phenotype cheaters, their presence in a group would decrease group fitness, since the number of extended phenotypes available for the group would decrease. This has been shown for biofilms produced by "altruistic" variants of Pseudomonas aeruginosa in which the presence of biofilm cheaters decreased group fitness (Popat et al., 2012). Nevertheless, the fact that we see situations in nature where "altruistic" and "selfish" variants co-exist in the same population suggests that other evolutionary forces act like the group selection scenario proposed by Sober and Wilson (1998).

While some aspects of the extended fitness hypothesis have been previously discussed, especially in the context of indirect genetic effects (Agrawal et al., 2001; Mutic and Wolf, 2007) and interacting phenotypes (Moore et al., 1997), the theoretical framework presented here is novel. The fact that extended phenotypes can be manipulated in controlled conditions provides a wide range of opportunities for the extended fitness hypothesis to be tested. An ultimate test for the hypothesis would be a situation in which extended phenotypes are made unavailable to other members of the group. It is predicted by the extended fitness hypothesis that the fitness of that group will be less when compared to a control group where the extended phenotype is available to all members.

\section{Conflicts of interest}

The author declares no conflict of interest.

\section{ACKNOWLEDGMENTS}

Research supported by Instituto de Bioinformática e Biotecnologia do Brasil. The author is indebted to Manyuan Long (University of Chicago) and Beatriz Stransky (UFRN) for critical review of the manuscript.

\section{REFERENCES}

Abbot P, Abe J, Alcock J, Alizon S, et al. (2011). Inclusive fitness theory and eusociality. Nature 471: E1-E4.

Agrawal AF, Brodie ED, III and Wade MJ (2001). On indirect genetic effects in structured populations. Am. Nat. 158: 308-323.

Bailey R, Schonrogge K, Cook JM, Melika G, et al. (2009). Host niches and defensive extended phenotypes structure parasitoid wasp communities. PLoS Biol. 7: e1000179. 
Bailey NW (2012). Evolutionary models of extended phenotypes. Trends Ecol. Evol. 27: 561-569.

Brockmann HJ, Grafen A and Dawkins R (1979). Evolutionarily stable nesting strategy in a digger wasp. J. Theor. Biol. 77: 473-496.

Darwin CR (1859). On the Origin of Species by Means of Natural Selection or the Preservation of Favored Races in the Struggle for Life. John Murray, London.

Dawkins R (1976). The Selfish Gene. Oxford University Press, Oxford.

Dawkins R (1982). The Extended Phenotype. Oxford University Press, Oxford.

Hamilton WD (1964a). The genetical evolution of social behaviour. I. J. Theor. Biol. 7: 1-16.

Hamilton WD (1964b). The genetical evolution of social behaviour. II. J. Theor. Biol. 7: 17-52.

Hunter P (2009). Extended phenotype redux. How far can the reach of genes extend in manipulating the environment of an organism? EMBO Rep. 10: 212-215.

Minter NJ, Franks NR and Brown KA (2012). Morphogenesis of an extended phenotype: four-dimensional ant nest architecture. J. R. Soc. Interface 9: 586-595.

Moore AJ, Brodie ED and Wolf JB (1997). Interacting phenotypes and the evolutionary process: I-direct and indirect genetic effects of social interactions. Evolution 51: 1352-1362.

Mutic JJ and Wolf JB (2007). Indirect genetic effects from ecological interactions in Arabidopsis thaliana. Mol. Ecol. 16: 2371-2381.

Nadell CD and Bassler BL (2011). A fitness trade-off between local competition and dispersal in Vibrio cholerae biofilms. Proc. Natl. Acad. Sci. U. S. A. 108: 14181-14185.

Nowak MA, Tarnita CE and Wilson EO (2010). The evolution of eusociality. Nature 466: 1057-1062.

Popat R, Crusz SA, Messina M, Williams P, et al. (2012). Quorum-sensing and cheating in bacterial biofilms. Proc. Biol. Sci. 279: 4765-4771.

Reed JM and Dobson AP (1993). Behavioural constraints and conservation biology: Conspecific attraction and recruitment. Trends Ecol. Evol. 8: 253-256.

Schaedelin FC and Taborsky M (2006). Mating crater of Cyathopharynx furcifer (Cichlidae) are individually characteristic extended phenotypes. Anim. Behav. 72: 753-761.

Schaedelin FC and Taborsky M (2009). Extended phenotypes as signals. Biol. Rev. Camb. Philos. Soc. 84: 293-313.

Schuck-Paim C and Alonso WJ (2001). Deciding where to settle: conspecific attraction and web-site selection in the orbweb spider Nephilengys cruentata. Anim. Behav. 62: 1007-1012.

Sober E and Wilson DS (1998). Unto Others: The Evolution and Psychology of Unselfish Behavior. Harvard University Press, Cambridge.

Xavier JB and Foster KR (2007). Cooperation and conflict in microbial biofilms. Proc. Natl. Acad. Sci. U. S. A. 104: 876-881. 